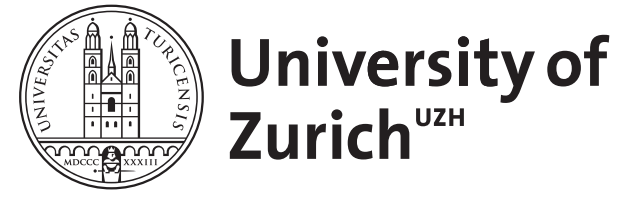

\title{
Models of triple covers
}

Kresch, Andrew ; Tschinkel, Yuri

DOI: https://doi.org/10.1134/s000143461905016x

Posted at the Zurich Open Repository and Archive, University of Zurich ZORA URL: https://doi.org/10.5167/uzh-174502

Journal Article

Accepted Version

Originally published at:

Kresch, Andrew; Tschinkel, Yuri (2019). Models of triple covers. Mathematical Notes, 105(5-6):795-797. DOI: https://doi.org/10.1134/s000143461905016x 


\title{
MODELS OF TRIPLE COVERS
}

\author{
ANDREW KRESCH AND YURI TSCHINKEL
}

\begin{abstract}
We exhibit, for a degree 3 covering of algebraic varieties, a model where the covering is a finite covering of smooth projective varieties branched over a smooth divisor.
\end{abstract}

\section{INTRODUCTION}

Let $k$ be a field of characteristic 0 . It is well known that any morphism of projective varieties $\psi: T \rightarrow S$ over $k$, that is generically finite of degree 2 , can be put into a commutative diagram

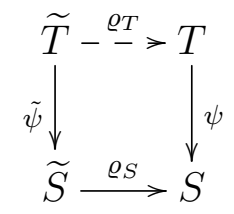

with smooth projective varieties $\widetilde{S}$ and $\widetilde{T}$, such that $\varrho_{S}$ is a birational morphism, $\varrho_{T}$ is a birational map, and $\tilde{\psi}$ is a degree 2 finite covering. The branch locus of a degree 2 finite covering of smooth projective varieties is a smooth divisor.

In this note, we establish an analogous theorem for triple covers, over perfect fields of characteristic not equal to 2 or 3 . Good models of triple covers are important for the construction of models of fibrations (over a base of arbitrary dimension), when the symmetry group of the geometric generic fiber admits the symmetric group $\mathfrak{S}_{3}$ as a quotient, as is the case for fibrations in sextic del Pezzo surfaces [1]-[4].

There is an extensive literature on triple covers of surfaces, e.g., [5][9]. In [8], a theorem similar to the one in this note is proved for triple covers of surfaces, by a method related to the classical solution of a cubic equation. Our approach is more geometric, and is based on an analysis of ramification in codimension 1 and 2 .

We produce $\tilde{\psi}: \widetilde{T} \rightarrow \widetilde{S}$, ramified over a smooth divisor of $\widetilde{S}$. By contrast, many of the (non-cyclic) degree 3 coverings of smooth projective varieties that occur naturally have singular branch locus, such as

Date: November 1, 2018. 
6-cuspidal sextic with cusps on a conic as branch locus of a general projection of a smooth cubic surface [10]. Such a covering is totally ramified (geometrically, only one pre-image in $T$ ) only over the cusps; after the procedure described here has been applied there is total ramification over entire components of the branch locus.

As explained in [11, Exa. 3.1], models of degree $\geq 4$ covers of surfaces as in (1) do not exist in general.

Acknowledgments: The second author was partially supported by NSF grant 1601912 and by the Laboratory of Mirror Symmetry NRU HSE, RF Government grant, ag. No. 14.641.31.0001.

\section{Finite COVERS AND RAMIFICATION}

Let $k$ be a field and $S$ a smooth variety over $k$, i.e., a separated geometrically integral scheme of finite type over $k$. By the normalization of $S$ in a finite field extension of $k(S)$ we have a canonical correspondence between finite field extensions of $k(S)$ and connected normal $k$-schemes with finite surjective morphism to $S$. The set of points where such a morphism $T \rightarrow S$ fails to be étale is a closed subset $Z \subset T$ which is

- equal to $T$ if and only the associated finite field extension of $k(S)$ is inseparable;

- is otherwise of pure codimension 1 or empty, by the ZariskiNagata purity theorem [12, Thm. X.3.1].

Consequently, if there exists a simple normal crossing divisor $D \subset S$, $D=D_{1} \cup \cdots \cup D_{n}$ with $D_{i}$ irreducible for all $i$, such that $T \times{ }_{S}(S \backslash D) \rightarrow S$ is étale, then the branch locus (the image of $Z$ in $S$ ) is of the form $\bigcup_{i \in I} D_{i}$ for some $I \subset\{1, \ldots, n\}$.

Once a finite field extension of $k(S)$ has been specified, when we refer to the branch locus we mean the branch locus of $T \rightarrow S$, where $T$ is the normalization of $S$ in the given field extension of $k(S)$.

\section{MAIN RESULT}

Let $k$ be a perfect field of characteristic not equal to 2 or $3, S$ a smooth projective variety over $k$, and $T$ a projective variety with morphism to $S$ that is generically finite of degree 3. If the field extension $k(T) / k(S)$ is cyclic, then an argument just as in the case of double covers yields a commutative diagram (1), where $\tilde{\psi}: \widetilde{T} \rightarrow \widetilde{S}$ is a cyclic degree 3 covering of smooth projective varieties branched over a smooth divisor on $\widetilde{S}$. As in the case of double covers, a form of resolution of singularities for divisors on $S$ is required; for instance, it is sufficient if the following is available: 
(R) embedded resolution of singularities of divisors on $S$ by iterated blow-up with smooth center.

Of course, this is available (Hironkaka) when $k$ has characteristic zero. When $k$ has positive characteristic, this is available for $\operatorname{dim}(S) \leq 3$ (trivial/classical for $\operatorname{dim}(S) \leq 2$, due to Abhyankar for $\operatorname{dim}(S)=3$ ).

Theorem 1. Let $k$ be a perfect field of characteristic not equal to 2 or 3, $S$ a smooth projective variety over $k$, and $\psi: T \rightarrow S$ a morphism of projective varieties that is generically finite of degree 3 . We suppose that $(\mathrm{R})$ holds for $S$. Then there exists a commutative diagram

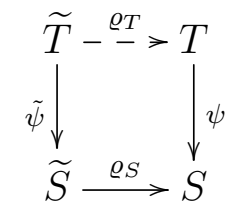

with smooth projective varieties $\widetilde{S}$ and $\widetilde{T}$, such that

- $\varrho_{S}$ is a birational morphism;

- $\varrho_{T}$ is a birational map;

- $\underset{\mathcal{\psi}}{\mathrm{S}}$ is a degree 3 finite covering, branched over a smooth divisor of $\widetilde{S}$.

Proof. As mentioned, when $k(T) / k(S)$ is cyclic this is achieved by applying $(\mathrm{R})$ to make the branch locus into a simple normal crossing divisor and repeatedly blowing up components of the intersection of a pair of components of the branch locus to make the branch locus smooth. Essential for the second step is the observation that a component $Y$ of an intersection $D_{i} \cap D_{j}(i \neq j)$ of components of the simple normal crossing branch locus $D=D_{1} \cup \cdots \cup D_{n}$ (achieved by the first step) may be assigned to one of two types, according to whether the branch locus for $k(T) / k(S)$ of the blow-up $B \ell_{Y} S$ has the exceptional divisor as a component. Let $\ell$, respectively $m$ denote the number of components $Y \subset D_{i} \cap D_{j}$ for some $i \neq j$, for which the exceptional divisor of $B \ell_{Y} S$ is, respectively is not, a component of the branch locus. (For simplicity of notation we continue to write $S$, even though the first step potentially makes a birational modification.) By blowing up some $Y$ for which the exceptional divisor is not a component of the branch locus whenever this is possible, and otherwise blowing up any $Y$, we obtain for $(\ell, m) \neq(0,0)$ a pair $\left(\ell^{\prime}, m^{\prime}\right)$ associated with $S^{\prime}:=B \ell_{Y} S$ that is smaller than $(\ell, m)$ in lexicographic order.

In the non-cyclic case we proceed with the same first step, making the branch locus into a simple normal crossing by applying (R). Now the branch locus has components of two kinds: some, say $D_{1} \cup \cdots \cup D_{n}$, with 
simple ramification and others, $D_{1}^{\prime} \cup \cdots \cup D_{n^{\prime}}^{\prime}$ with total ramification. We adopt the convention that the $D_{i}$ and $D_{i^{\prime}}^{\prime}$ are all irreducible.

The discriminant of $k(T) / k(S)$ determines a quadratic extension of $k(S)$ with branch locus $D_{1} \cup \cdots \cup D_{n}$. By blowing up intersections of pairs of components, we achieve $D_{i} \cap D_{j}=\emptyset$ for $i \neq j$.

We claim that $D_{i} \cap D_{i^{\prime}}^{\prime}=\emptyset$ for all $i$ and $i^{\prime}$. This follows from the fact that the pre-image of $D_{i^{\prime}}^{\prime}$ in the discriminant double cover would be irreducible. We argue by contradiction, replacing $S$ by a strict henselization of the local ring of $S$ at the generic point of a component of $D_{i} \cap D_{i^{\prime}}^{\prime}$. We still have a cubic extension of the residue field of the generic point, still with nontrivial discriminant, and this yields a cyclic cubic extension of the discriminant cover. This must be obtained from a cyclic cubic extension below by base change to the double cover, and we have a contradiction.

We may have $D_{i^{\prime}}^{\prime} \cap D_{j^{\prime}}^{\prime} \neq \emptyset$ for some $i^{\prime} \neq j^{\prime}$, but then as described at the beginning of the proof we may deal with this by blowing up components of intersections $D_{i^{\prime}}^{\prime} \cap D_{j^{\prime}}^{\prime}$. Then we have a smooth branch locus and hence a smooth model of the covering.

\section{REFERENCES}

[1] J.-L. Colliot-Thélène, N. A. Karpenko, and A. S. Merkurjev, Rational surfaces and the canonical dimension of the group $\mathrm{PGL}_{6}$, Algebra $i$ Analiz, 19(5):159-178, 2007.

[2] M. Blunk, Del Pezzo surfaces of degree 6 over an arbitrary field. J. Algebra, $323(1): 42-58,2010$.

[3] N. Addington, B. Hassett, Yu. Tschinkel, and A. Várilly-Alvarado, Cubic fourfolds fibered in sextic del Pezzo surfaces, arXiv:1606.05321.

[4] A. Kuznetsov, Derived categories of families of sextic del Pezzo surfaces, arXiv: 1708.00522.

[5] P. du Val, On triple planes having branch curves of order not greater than twelve, J. London Math. Soc., 8(3):199-206, 1933.

[6] R. Miranda, Triple covers in algebraic geometry, Amer. J. Math., 107(5):1123$1158,1985$.

[7] H. Tokunaga, Triple coverings of algebraic surfaces according to the Cardano formula, J. Math. Kyoto Univ., 31(2):359-375, 1991.

[8] S.-L. Tan. Triple covers on smooth algebraic varieties, Geometry and nonlinear partial differential equations (Hangzhou, 2001), AMS/IP Stud. Adv. Math., 29, Amer. Math. Soc., Providence, RI, 2002, 143-164.

[9] T. Shirane, A note on normal triple covers over $\mathbf{P}^{2}$ with branch divisors of degree 6, Kodai Math. J., 37(2):330-340, 2014.

[10] O. Zariski, On the problem of existence of algebraic functions of two variables possessing a given branch curve, Amer. J. Math., 51(2):305-328, 1929.

[11] T. Shirane, On 4-fold covers of algebraic surfaces, Kyushu J. Math., 64(2):297322,2010 
[12] A. Grothendieck. Revêtements étales et groupe fondamental (SGA 1), SpringerVerlag, Berlin, 1971.

Institut für MAthematik, Universität Zürich, Winterthurerstrasse 190, CH-8057 ZÜRICH, SwitzerLAND

Email address: andrew.kresch@math.uzh.ch

Courant Institute, 251 Mercer Street, New York, NY 10012, USA

Simons Foundation, 160 Fifth Av., New York, NY 10010, USA

Email address: tschinkel@cims.nyu.edu 